\title{
Dynamic response of a thin sessile drop of conductive liquid to an abruptly applied or removed electric field
}

\author{
L. T. Corson, N. J. Mottram, B. R. Duffy, and S. K. Wilson* \\ Department of Mathematics \& Statistics, University of Strathclyde, 26 Richmond Street, Glasgow G1 1XH, United Kingdom \\ C. Tsakonas and C. V. Brown \\ School of Science \& Technology, Nottingham Trent University, Clifton Lane, Nottingham NG11 8NS, United Kingdom
}

(Received 31 May 2016; published 24 October 2016)

\begin{abstract}
We consider, both theoretically and experimentally, a thin sessile drop of conductive liquid that rests on the lower plate of a parallel-plate capacitor. We derive analytical expressions for both the initial deformation and the relaxation dynamics of the drop as the electric field is either abruptly applied or abruptly removed, as functions of the geometrical, electrical, and material parameters, and investigate the ranges of validity of these expressions by comparison with full numerical simulations. These expressions provide a reasonable description of the experimentally measured dynamic response of a drop of conductive ionic liquid 1-butyl-3-methyl imidazolium tetrafluoroborate.
\end{abstract}

DOI: 10.1103/PhysRevE.94.043112

\section{INTRODUCTION}

The deformation of films and drops of liquid due to an externally applied electric field is both a scientifically interesting and a practically important problem that has been studied both experimentally and theoretically for well over a century. Examples of this literature include the pioneering work by Swan [1], in which the surface of a resin film was destabilized by an electric field, the work on drops by Cheng and Miksis [2], Basaran and Scriven [3], Wohlhuter and Basaran [4], Berge and Peseux [5], Quilliet and Berge [6], Reznik et al. [7], Mugele and Baret [8], Chen and Bonaccurso [9], Corson et al. [10], Tsakonas et al. [11], and the references therein, and Sec. III.C of the review article by Craster and Matar [12]. The resulting deformation and changes in morphology of the liquid are of interest both because they can give insight into the fundamentals of wetting and de-wetting phenomena and because of their importance in a number of practical engineering applications. In particular, a parallel-plate capacitor geometry consisting of two parallel charged conducting plates with an air gap between them has been widely used to manipulate both films and drops of liquid. For example, Schaffer et al. [13,14], Morariu et al. [15], Klingner et al. [16,17], Pease and Russel [18], Craster and Matar [19], Merkt et al. [20], Verma et al. [21], Wu et al. [22], Tseluiko et al. [23], Yeoh et al. [24], Manigandan et al. [25], Berendsen et al. [26], Ramkrishnan and Kumar [27], and Corbett and Kumar [28] used electric fields to study the stability of and pattern formation in liquid films on uniform substrates and/or to generate prescribed patterns on nonuniform substrates. The latter is of particular interest as it enables manufacturers to replicate small-scale electrode patterns in a liquid film (which can then be "frozen

\footnotetext{
*s.k.wilson@strath.ac.uk
}

Published by the American Physical Society under the terms of the Creative Commons Attribution 3.0 License. Further distribution of this work must maintain attribution to the author(s) and the published article's title, journal citation, and DOI. in" by solidifying the film, i.e., electrostatic lithography). In addition, Yeo et al. [29] studied the manipulation and "surgery" of a thin drop using a nonuniform electric field.

In the present work we consider a thin sessile drop of conductive liquid that rests on the lower plate of a parallel-plate capacitor. This lower capacitor plate consists of an electrode whose upper surface is coated with a surface-treatment agent to achieve a low contact angle with contact-line pinning, which will be termed the substrate. The upper capacitor plate consists of an uncoated electrode, which will be termed the upper electrode. The liquid forms a thin drop partially coating the substrate. In this work, the dynamic response of the drop to the application or removal of a DC voltage difference across the capacitor, in particular, the dependence of the response on the geometrical, electrical, and material parameters, is considered both theoretically and experimentally. A theoretical description of the experiment is given in Sec. II, with the details of the experimental procedure given in Sec. III.

\section{THEORETICAL MODEL}

To complement the experimental investigations described in Sec. III, we consider a theoretical model of the system in order to understand better both its static and its dynamic behavior. The geometry of the system is shown in Fig. 1. We assume that the drop is rotationally symmetric about the vertical axis and hence has a circular contact line with the substrate. We also assume that the liquid is incompressible and perfectly conductive and that the region above the drop inside the capacitor is filled with air, which we take to be a perfectly insulating fluid. Based on the experimental observations we assume that the contact line is pinned so that, for any applied electric field and for all time, the base radius of the drop remains constant, and is denoted by $R$. We use cylindrical polar coordinates with origin on the substrate at the center of the base of the drop and $z$ axis along the normal to the capacitor plates, as shown in Fig. 1(a). The dependent variables in this model are, therefore, the position of the drop free surface (the liquid-air interface), which here we specify by its height above the substrate, $z=h(r, t)$, the liquid pressure $p(r, z, t)$ and velocity $\mathbf{u}(r, z, t)$, 
(a)

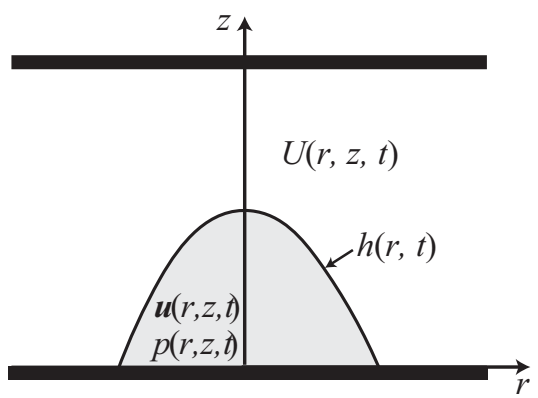

(b)

(c)

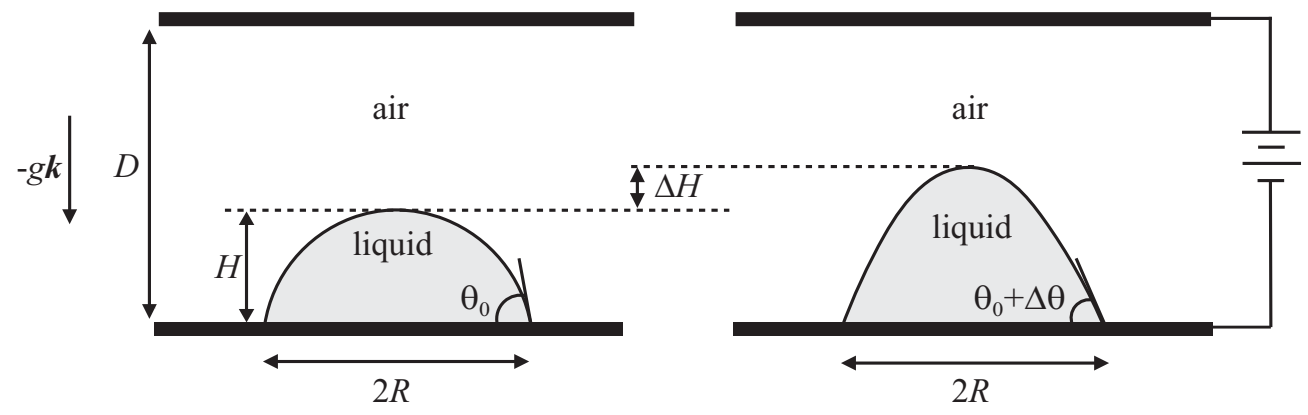

FIG. 1. The geometry of a sessile drop resting on the lower plate of a parallel-plate capacitor. (All labels are described in Sec. II.) (a) Definition of the cylindrical polar coordinates $r$ and $z$ and the dependent variables used in the model. (b) Drop with no electric field applied. (c) Drop deformed by the application of a DC voltage across the capacitor.

and the electric potential $U(r, z, t)$ in the drop and the air gap. In this model we assume that the air is a passive gas with zero density, so that the pressure in the air is equal to the constant atmospheric pressure $p_{\mathrm{a}}$, and that the velocity of the air is zero.

In equilibrium and with no applied electric field the shape of the drop free surface is determined by a static balance between surface tension and gravity, the latter of which acts in the negative $z$ direction, denoted by $-\mathbf{k}$. For this zero-field equilibrium situation we denote the height of the drop apex above the substrate by $H$, and the contact angle by $\theta_{0}$, as shown in Fig. 1(b). The electrodes of the capacitor are separated by a constant distance $D$. At the lower electrode the electric potential is held at zero for all time, and an electric field is applied across the capacitor by imposing a time-dependent voltage at the upper electrode, denoted by $V(t)$. The application of the electric field causes a transient deformation of the drop, resulting in a time-dependent apex height of $h(0, t)=H+\Delta H(t)$ and a time-dependent contact angle of $\theta(t)=\theta_{0}+\Delta \theta(t)$, as shown in Fig. 1(c).

\section{A. Governing equations}

In both equilibrium and during the transient deformation of the drop, the dependent variables will be governed by the Gauss and Faraday laws for electrostatics, flow equations representing the conservation of mass and the balance of linear momentum, and boundary conditions that couple the variables through the stress balance and kinematic conditions at the drop free surface.

Since the drop is assumed to be of a perfectly conductive liquid, the electric potential inside the drop is uniform. In the air gap we assume that there is no induced magnetic field, so that Faraday's law for the electric field $\mathbf{E}$ becomes $\boldsymbol{\nabla} \times \mathbf{E}=\mathbf{0}$ and we may therefore define the electric potential $U$ according to $\mathbf{E}=-\nabla U$. We also assume that there are no free charges in the air, so that Gauss's law is

$$
-\nabla \cdot \mathbf{E}=\nabla^{2} U=0 .
$$

Since the drop is in contact with the substrate, which is at zero potential, the electric potential within the drop is therefore equal to zero.

The electric potential in the air, which is governed by Eq. (1), is subject to boundary conditions at the upper surface of the substrate, at the upper electrode, and at the drop free surface, namely

$$
\begin{gathered}
U(r, 0, t)=0, \\
U(r, D, t)=V(t), \\
U(r, h, t)=0 .
\end{gathered}
$$

The velocity inside the drop, i.e., for $0<z<h(r, t)$, denoted by $\mathbf{u}=(u(r, z, t), 0, w(r, z, t))$, where $u(r, z, t)$ and $w(r, z, t)$ are the velocity components in the $r$ and $z$ directions, respectively, and the pressure $p(r, z, t)$ are governed by the mass and momentum balances

$$
\begin{gathered}
\nabla \cdot \mathbf{u}=0, \\
\rho \frac{\partial \mathbf{u}}{\partial t}+\rho(\mathbf{u} \cdot \nabla) \mathbf{u}=-\nabla p+\mu \nabla^{2} \mathbf{u}-\rho g \mathbf{k},
\end{gathered}
$$

where $\mu$ is the constant liquid dynamic viscosity, $\rho$ is the constant liquid density, and $g$ is the magnitude of the acceleration due to gravity. 
As well as the flow equations for the velocity and pressure in the bulk of the drop, boundary conditions must also be specified at $z=0$ and $z=h(r, t)$. At the lower boundary of the drop, i.e., $z=0$ for $0 \leqslant r \leqslant R$, a no-slip and no-penetration condition ensures the velocity is zero, $\mathbf{u}(r, 0, t)=\mathbf{0}$, and at the drop free surface (i.e., $z=h$ for $0 \leqslant r \leqslant R$ ) the appropriate conditions are balances of normal and tangential stresses. In general, the stress tensor $\boldsymbol{\tau}$ has contributions due to pressure, viscous effects and dielectric effects. However, viscous effects are present only in the drop, and dielectric effects are present only in the air, so that the $i j$ th component of the stress tensor is given by

$$
\begin{gathered}
\tau_{i j}=-p \delta_{i j}+\mu\left(\frac{\partial u_{i}}{\partial x_{j}}+\frac{\partial u_{j}}{\partial x_{i}}\right), \\
\tau_{i j}=-p_{\mathrm{a}} \delta_{i j}+\epsilon_{0} \epsilon_{\mathrm{a}}\left(E_{i} E_{j}-\frac{1}{2}|\mathbf{E}|^{2} \delta_{i j}\right),
\end{gathered}
$$

inside and outside the drop, respectively. Here $\delta_{i j}$ is the Kronecker delta and $\epsilon_{0}=8.854 \times 10^{-12} \mathrm{Fm}^{-1}$ is the permittivity of free space. The permittivity of air is sufficiently close to that of free space that we can reasonably assume that the relative permittivity $\epsilon_{\mathrm{a}}=1$, although we will retain the more general expression for the stress tensor in the air so that the model may also be applied to other systems in which the passive gas has $\epsilon_{\mathrm{a}} \neq 1$. Using these forms of the stress tensor we may then balance the difference in normal stress across the drop free surface with the capillary pressure to obtain

$$
[\mathbf{n} \cdot \boldsymbol{\tau} \cdot \mathbf{n}]_{1}^{2}=\sigma \nabla \cdot \mathbf{n},
$$

where we have used the notation $[\cdot]_{1}^{2}$ for the difference in a quantity across the interface, with 1 and 2 denoting the drop and air regions, respectively, $\sigma$ is the constant surface tension, and $\boldsymbol{\nabla} \cdot \mathbf{n}$ is twice the mean curvature of the drop free surface, for which the outward unit normal is

$$
\mathbf{n}=-\left[\frac{\partial h / \partial r}{\sqrt{1+(\partial h / \partial r)^{2}}}\right] \mathbf{r}+\left[\frac{1}{\sqrt{1+(\partial h / \partial r)^{2}}}\right] \mathbf{k},
$$

where $\mathbf{r}$ is the unit vector in the radial direction. Additionally, the balance of tangential stress at the drop free surface requires that

$$
[\mathbf{n} \cdot \boldsymbol{\tau} \cdot \mathbf{t}]_{1}^{2}=0 .
$$

In addition to these conditions on the stress tensor at the drop free surface, the kinematic condition at the free surface, conditions of a fixed contact line at $r=R$, and appropriate symmetry and smoothness conditions at $r=0$ must be satisfied:

$$
\begin{gathered}
\frac{\partial h}{\partial t}+u(r, h, t) \frac{\partial h}{\partial r}-w(r, h, t)=0, \\
h(R, t)=0, \quad \frac{\partial h}{\partial r}(0, t)=0, \quad \frac{\partial^{3} h}{\partial r^{3}}(0, t)=0 .
\end{gathered}
$$

The final constraint on the system is that the volume of the drop, given by

$$
2 \pi \int_{0}^{R} r h(r, t) d r
$$

remains constant for all time.
The type of model described by the differential equations $(1,5,6,12)$, with boundary conditions $(2,3,4,9,11,13)$ and volume constraint (14), is a relatively standard one for electric-field-induced drop deformation and flow. For example, a similar model was used by Craster and Matar [19], although they neglected gravity (because their typical film thicknesses are much smaller than we consider in the present work), treated infinite layers rather than a finite radius drop, and considered the case of a leaky dielectric layer, where ionic charge accumulates at the interface. As we will demonstrate below, gravity is not negligible in the present experiments because of the relatively large size of the drops considered; moreover, a perfectly conductive liquid model, with no interfacial charge accumulation, provides a reasonable description of the present experimental results.

\section{B. Nondimensionalization}

Our model contains three independent variables, namely the coordinates $r$ and $z$ and time $t$, and five dependent variables, namely the velocities $u(r, z, t)$ and $w(r, z, t)$, the pressure $p(r, z, t)$, the electric potential $U(r, z, t)$, and the drop height $h(r, t)$. There are also the geometrical parameters $d, D, H$, and $R$, the material parameters $\epsilon_{\mathrm{a}}, \mu, \rho$, and $\sigma$, and the additional parameters associated with the applied voltage $V(t)$, gravity $g$, atmospheric pressure $p_{\mathrm{a}}$, and the permittivity of free space $\epsilon_{0}$. However, as usual, the number of independent parameters in the model can be reduced through nondimensionalization of the governing equations and boundary conditions. By rescaling horizontal lengths with the radius $R$, vertical lengths with the zero-field drop apex height $H$, the contact angle with the zero-field drop aspect ratio $H / R$, time with the timescale associated with the classical surface-tension-driven leveling of a viscous film, namely $\mu R^{4} / \sigma H^{3}$, liquid velocities with the appropriate speeds for this timescale, the pressure with the appropriate pressure due to surface tension, and the electric potentials with a typical applied voltage $V_{\mathrm{m}}$, we write

$$
\begin{aligned}
r & =R r^{*}, \quad z=H z^{*}, \quad t=\frac{\mu R^{4}}{\sigma H^{3}} t^{*}, \\
h & =H h^{*}, \quad \theta=\frac{H}{R} \theta^{*}, \\
u & =\frac{\sigma H^{3}}{\mu R^{3}} u^{*}, \quad w=\frac{\sigma H^{4}}{\mu R^{4}} w^{*}, \quad p=\frac{\sigma H}{R^{2}} p^{*}, \\
U & =V_{\mathrm{m}} U^{*}, \quad V=V_{\mathrm{m}} V^{*} .
\end{aligned}
$$

This nondimensionalization reduces the number of independent parameters in the model to just four: an appropriate nondimensional electric Bond number, $B$, measuring the relative size of the electric field and surface-tension effects at the drop free surface, a gravitational Bond number, $G$, measuring the relative size of gravity and surface-tension effects, a scaled cell gap $d$, and a drop aspect ratio $\delta$, given by

$$
B=\frac{\epsilon_{0} \epsilon_{\mathrm{a}} V_{\mathrm{m}}^{2} R^{2}}{\sigma H D^{2}}, \quad G=\frac{\rho g R^{2}}{\sigma}, \quad d=\frac{D}{H}, \quad \delta=\frac{H}{R},
$$

respectively. In the present experiments the contact angle and the aspect ratio $\delta$ were small in equilibrium and remained small for all values of the applied voltage. An appropriate additional simplification of this model is therefore to consider 
the classical thin-film approximation, expanding all dependent variables in powers of $\delta^{2} \ll 1$ and retaining only leading order terms. Note, however, that for the present experiments, the scaled cell gap is not small and so we assume that $d=O(1)$.

Because of the nondimensionalization we have employed, the inertial terms in the balance-of-momentum equations are multiplied by a reduced Reynolds number, $\delta^{4} \sigma \rho H / \mu^{2}$. Hence assuming that $\delta^{4} \sigma \rho H / \mu^{2} \ll 1$ we may neglect inertial effects at leading order, and the Reynolds number does not enter the present model. With this approach the leading order terms in the mass and momentum balance equations (5) and (6) are, with the stars dropped for clarity,

$$
\begin{gathered}
\frac{1}{r} \frac{\partial}{\partial r}(r u)+\frac{\partial w}{\partial z}=0, \\
\frac{\partial p}{\partial r}-\frac{\partial^{2} u}{\partial z^{2}}=0, \\
\frac{\partial p}{\partial z}+G=0,
\end{gathered}
$$

and from Gauss's law (1) the electric potential $U$ must satisfy

$$
\frac{\partial^{2} U}{\partial z^{2}}=0
$$

These equations are to be solved subject to the boundary conditions

$$
\begin{gathered}
u(r, 0, t)=0, \quad w(r, 0, t)=0, \\
\frac{\partial h}{\partial t}+u(r, h, t) \frac{\partial h}{\partial r}-w(r, h, t)=0, \\
U(r, 0, t)=0, \quad U(r, h(r, t), t)=0, \quad U(r, d, t)=V(t), \\
p(r, h(r, t), t)-p_{\mathrm{a}}+\frac{B d^{2}}{2}\left(\frac{\partial U}{\partial z}(r, h(r, t), t)\right)^{2} \\
=-\frac{1}{r} \frac{\partial}{\partial r}\left(r \frac{\partial h}{\partial r}\right), \\
\frac{\partial u}{\partial z}(r, h(r, t), t)=0, \\
\frac{\partial h}{\partial r}(0, t)=0, \quad \frac{\partial^{3} h}{\partial r^{3}}(0, t)=0 .
\end{gathered}
$$

In the special case of a drop in equilibrium $(u \equiv 0, w \equiv 0)$ with no electric field ( $V \equiv 0, U \equiv 0$ ) the governing equations reduce to a balance between gravity and surface-tension effects, namely

$$
\frac{d}{d r}\left[\frac{1}{r} \frac{d}{d r}\left(r \frac{d h}{d r}\right)\right]-G \frac{d h}{d r}=0 .
$$

The solution of this equation subject to the boundary conditions (26) is

$$
h(r)=\frac{I_{0}(\sqrt{G})-I_{0}(\sqrt{G} r)}{I_{0}(\sqrt{G})-1},
$$

where $I_{0}(\cdot)$ is the modified Bessel function of the first kind of order zero, corresponding to a drop with apex height $h(0)=1$, contact angle $\theta_{0}$, where

$$
\theta_{0}=\frac{\sqrt{G} I_{1}(\sqrt{G})}{I_{0}(\sqrt{G})-1}
$$

and volume

$$
\frac{\sqrt{G} I_{0}(\sqrt{G})-2 I_{1}(\sqrt{G})}{2 \sqrt{G}\left(I_{0}(\sqrt{G})-1\right)}
$$

where $I_{1}(\cdot)$ is the modified Bessel function of the first kind of order one.

\section{Evolution equation}

In this section we show that Eqs. (17)-(26) and (30) can be reduced to a single partial differential equation governing the evolution of the drop height $h(r, t)$ together with the boundary conditions (26) and the volume constraint

$$
\int_{0}^{1} r h(r, t) d r=\frac{\sqrt{G} I_{0}(\sqrt{G})-2 I_{1}(\sqrt{G})}{2 \sqrt{G}\left(I_{0}(\sqrt{G})-1\right)} .
$$

Equation (19) gives $p(r, z, t)=p_{\mathrm{a}}-G z+P(r, t)$, where $P(r, t)$ is called the modified pressure. With this solution for $p$, integration of Eq. (18) subject to Eq. (25) and the first of Eq. (21) then leads to the horizontal velocity:

$$
u=\frac{\partial P}{\partial r}\left(\frac{z^{2}}{2}-h z\right) .
$$

Using this solution for $u$, integrating Eq. (17) and using the second of Eq. (21) provides the vertical velocity:

$$
w=\frac{1}{6 r} \frac{\partial}{\partial r}\left[r z^{2}(z-3 h) \frac{\partial P}{\partial r}\right],
$$

and then Eq. (22) becomes

$$
\frac{\partial h}{\partial t}-\frac{1}{r} \frac{\partial}{\partial r}\left[\frac{r h^{3}}{3}\left(\frac{\partial P}{\partial r}\right)\right]=0 .
$$

Integration of Eq. (20) subject to the boundary condition (23) gives a linear electric potential within the air gap:

$$
U=V \times \begin{cases}\frac{z-h}{d-h} & \text { for }|r| \leqslant R \\ \frac{z}{d} & \text { for }|r|>R\end{cases}
$$

so that imposing the normal stress balance (24) leads to the solution for the modified pressure:

$$
P=G h-B \frac{V^{2} d^{2}}{2(d-h)^{2}}-\frac{1}{r} \frac{\partial}{\partial r}\left(r \frac{\partial h}{\partial r}\right) .
$$

Substituting this solution for $P$ into Eq. (34) leads to the partial differential equation for the evolution of the drop free 
surface, namely,

$$
\begin{gathered}
\frac{\partial h}{\partial t}+\frac{1}{r} \frac{\partial}{\partial r}\left(\frac { r h ^ { 3 } } { 3 } \left\{\frac{\partial}{\partial r}\left[\frac{1}{r} \frac{\partial}{\partial r}\left(r \frac{\partial h}{\partial r}\right)\right]\right.\right. \\
\left.\left.-G \frac{\partial h}{\partial r}+B \frac{V(t)^{2} d^{2}}{(d-h)^{3}} \frac{\partial h}{\partial r}\right\}\right)=0,
\end{gathered}
$$

subject to the boundary conditions (26) and the volume constraint (31).

In the present work we will consider both static and dynamic solutions of Eq. (37) subject to Eqs. (26) and (31) using both numerical and approximate analytical methods. We will then compare these theoretical predictions with experimental measurements of the drop deformation and of the dynamics using the drop apex height difference as a measure. The (nondimensional) drop apex height difference is the difference between the deformed drop apex height and the zero-field equilibrium drop apex height at $r=0$, given by

$$
\Delta H(t)=h(0, t)-1 .
$$

When comparing different theoretical approaches we will also consider the corresponding difference in the contact angle, given by

$$
\Delta \theta(t)=-\left.\frac{\partial h}{\partial r}\right|_{r=1}-\theta_{0},
$$

where the zero-field equilibrium contact angle $\theta_{0}$ is given by Eq. (29).

\section{EXPERIMENTAL PROCEDURE}

Achieving the experimental geometry outlined in Sec. II and illustrated in Fig. 1 was challenging because of the necessity simultaneously to ensure a low contact angle drop with a pinned and circular contact line, and to maintain sufficient parallelism between the substrate and the upper electrode to maintain a cylindrically symmetric drop when the voltage was applied.

The capacitor plates consisted of two soda lime glass slides with continuous layers of the transparent conductor indium tin oxide (100 Ohm/square, $25 \mathrm{~nm}$ thickness, Praezisions Glas and Optik GmbH, Iserlohn, Germany) on their inner surfaces to form the capacitor electrodes. This allowed simultaneous optical imaging of the drop both from the side and from above (i.e., through the upper capacitor plate) during the course of the experiment. The electrode on the lower capacitor plate was coated with a surface-treatment agent, namely polyvinyl alcohol (PVA, molecular weight of 77000-79000 atomic mass units), to achieve a low contact angle with contact-line pinning. This coating was produced by spin-coating (at 3000 revolutions per minute) from a $0.5 \mathrm{wt} \%$ solution of the PVA in deionized water, allowing the plate to dry under a nitrogen gas flow, and then treating it in a UV ozone box (Bioforce Nanosciences, $185 \mathrm{~nm}$ and $254 \mathrm{~nm}$ spectral lines, $4.6 \mathrm{~mW} / \mathrm{cm}^{2}$ at $254 \mathrm{~nm}$ and $5 \mathrm{~cm}$ lamp-to-substrate distance) for $3 \mathrm{~s}$.

A sessile drop of the conductive ionic liquid 1-butyl-3methyl imidazolium tetrafluoroborate $\left([\mathrm{Bmim}]\left[\mathrm{BF}_{4}\right]\right.$, SigmaAldrich) was dispensed onto the substrate. The PVA coating (which was significantly less than $100 \mathrm{~nm}$ thick and was not continuous, with a significant density of pinholes as a result of the processing conditions) provided a low-energy surface for the substrate on which the circular contact line spreads to form the drop. It is likely that the roughness of the indium tin oxide layer (which was not significantly smoothed by the PVA coating in our experiments) in combination with inhomogeneities introduced by the PVA coating itself and the subsequent UV ozone treatment all played a role in the subsequent pinning of the contact line observed throughout the present experiments. In order to reduce differences between experimental runs, the same drop was used for all of the equilibrium and dynamic measurements. The equilibrium height, radius, and contact angle values of the drop used in the present experiment were $H_{\exp }=(0.141 \pm 0.01) \times 10^{-3} \mathrm{~m}$, $R_{\text {exp }}=(1.815 \pm 0.004) \times 10^{-3} \mathrm{~m}$, and $\theta_{\exp }=(9.2 \pm 0.4)^{\circ}$, respectively. Experimental images of the drop with no applied voltage and with an applied voltage of $865 \mathrm{~V}$ are shown on Figs. 2(a) and 2(b), respectively.

The upper plate was moved into position on an XYZ translation stage to give a maximum air gap between the upper electrode and the substrate of $D=(0.544 \pm 0.006) \times 10^{-3}$ $\mathrm{m}$. An additional two-axis translation and rotation stage for the substrate was used to ensure that the plates were parallel. DC voltage pulses were applied to the upper electrode using a waveform generator (TGA1244, Aim \& Thurlby Thandar Instruments) connected to a high-slew-rate voltage amplifier (PZD700A, Trek Inc.). The drop was illuminated by an ultrahigh-brightness single white LED source. Images of the drop were recorded using a high-speed camera (HHC X4, Mega Speed Corp.) at 2009 frames per second. Using a Simulation Program with Integrated Circuit Emphasis (SPICE) model we have confirmed that the RC transients in our experiment are several orders of magnitude smaller than the time resolution used. Using a $\times 5$ objective lens with a $25 \mathrm{~mm}$ lens extension tube allowed images of the whole drop (and, in particular, the drop diameter) to be recorded with a resolution of (3.84 \pm $0.02) \times 10^{-6} \mathrm{~m}$ per pixel in the image. A $200 \mathrm{~mm}$ extension tube was used to record higher-magnification time-dependent images with resolution of $(1.11 \pm 0.03) \times 10^{-6} \mathrm{~m}$ per image pixel of the drop apex as it moved in response to the voltage pulse. The camera was focused on the apex of the drop so that very small changes in the height could be detected. In order to optimize the accuracy of the high resolution experimental data obtained, the apparatus was mounted on a vibration isolation sheet, and translation and mounting stages were firmly anchored and weighted to dampen vibrations. Despite these measures, some frame-rate-dependent aliasing effects from vibrations from the camera fan were unavoidable, particularly for small height changes at lower voltages, in addition to some inevitable scatter and pixel-discretization noise. To ameliorate these effects the drop height was tracked using image enhancement alongside polynomial fitting of the drop apex shape, and an 80-point rolling time average was used to smooth the data.

The conductive liquid used, namely $[\mathrm{Bmim}]\left[\mathrm{BF}_{4}\right]$, has a low vapour pressure and so shows negligible evaporation during the experiments [30-32], consistent with the assumption of constant volume used in Sec. II A. Since the density, viscosity, and conductivity of imidazolium-based ionic liquids depend on hydration as well as temperature [33-35], the experiments were performed in a temperature-controlled environment at 

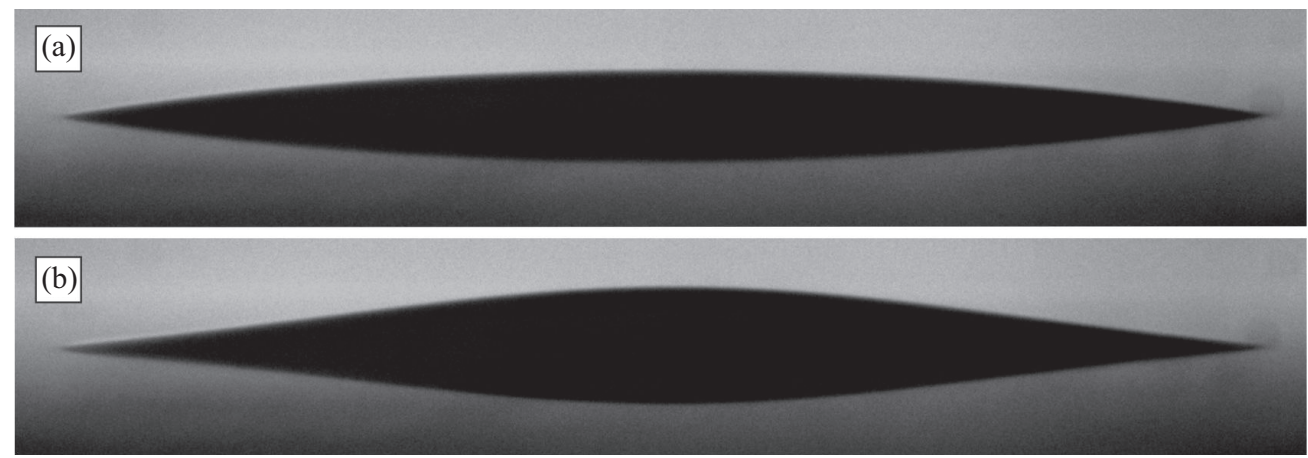

FIG. 2. Experimental images of the thin $[\mathrm{Bmim}]\left[\mathrm{BF}_{4}\right]$ drop with (a) no applied voltage and (b) an applied voltage of $865 \mathrm{~V}$, also showing its reflection in the substrate on which it rests within the parallel-plate capacitor.

$20^{\circ} \mathrm{C}$ with the relative humidity in the narrow range between $50 \%$ and $55 \%$. When initially dispensed, $[\mathrm{Bmim}]\left[\mathrm{BF}_{4}\right]$ is hygroscopic, and so the drop was left for over a week to reach equilibrium hydration before the experiments began. The key physical properties of the liquid, measured under the ambient conditions of the experiment, were as follows: surface tension $\sigma_{\exp }=(40.9 \pm 0.5) \times 10^{-3} \mathrm{~N} \mathrm{~m}^{-1}$, obtained using the pendant drop method [36] with Drop Shape Analysis (A. Krüss Optronic GmbH, Hamburg, Germany); viscosity $\mu_{\exp }=(58 \pm 3) \times 10^{-3}$ Pa s, obtained using a CSL2 TA Instruments rheometer; and density $\rho_{\exp }=1200 \pm 10$ $\mathrm{kg} \mathrm{m} \mathrm{m}^{-3}$, obtained by comparing the weight of a capillary tube filled with $[\mathrm{Bmim}]\left[\mathrm{BF}_{4}\right]$ with the same tube filled with distilled water. The nondimensional parameters are therefore $B_{\exp }=0.1710 \times 10^{-4} V_{\mathrm{m}}^{2}, G_{\exp }=0.9472, d_{\exp }=3.857$, $\delta_{\exp }=0.0777$, and one unit of nondimensional time is equal to $5.490 \mathrm{~s}$. In the present experiment voltages between zero and $907 \mathrm{~V}$ were used, corresponding to electric Bond numbers from zero to 14.0673 .

\section{COMPARISONS BETWEEN EXPERIMENT AND THEORY}

In this section we present comparisons between the experimental measurements and numerical and approximate analytical solutions for both the equilibrium value and the dynamic behavior of the drop apex height difference $\Delta H$. We consider the equilibrium drop apex height difference as a function of the applied DC voltage, demonstrating that the approximations and assumptions used in the model are valid for sufficiently small values of the electric Bond number $B$. We then consider the dynamic drop apex height difference, comparing experimental results and numerical solutions in the case when the applied voltage changes abruptly. By appropriately linearizing the evolution equation for the drop apex height we are then able to derive analytical expressions for the timescales of the evolution of the drop shortly after the voltage is either abruptly switched on or abruptly switched off.

\section{A. Equilibrium solutions}

When, with a DC electric field applied so that $V(t) \equiv 1$, sufficient time is allowed for the drop to reach equilibrium, the present model simplifies and the drop free surface is governed by the static version of Eq. (37), namely

$$
\begin{aligned}
& \frac{d}{d r}\left(\frac{r h_{\mathrm{s}}^{3}}{3}\left\{\frac{d}{d r}\left[\frac{1}{r} \frac{d}{d r}\left(r \frac{d h_{\mathrm{s}}}{d r}\right)\right]-G \frac{d h_{\mathrm{s}}}{d r}+B \frac{d^{2}}{\left(d-h_{\mathrm{s}}\right)^{3}} \frac{d h_{\mathrm{s}}}{d r}\right\}\right) \\
& \quad=0
\end{aligned}
$$

subject to the boundary conditions (26) and the volume constraint (31). Here, we have used $h_{\mathrm{s}}(r)$ to denote the equilibrium solution for the drop free surface shape and will use $\theta_{\mathrm{s}}$ to denote its contact angle.

For the case of zero field, $B=0$, the solution for $h_{\mathrm{s}}(r)$, which we denote as $h_{\mathrm{s} 0}(r)$, has already been given in Eq. (28).

For the case of nonzero field, $B \neq 0$, we may solve Eq. (40) numerically, but no analytical progress has been made without assuming that the electric Bond number is small, $B \ll 1$, and that gravity is negligible, $G=0$. If we make these assumptions then we can expand the solution of Eq. (40) for $h_{\mathrm{s}}(r)$ in powers of $B$ :

$$
\left.h_{\mathrm{s}}(r)\right|_{G=0}=1-r^{2}+\left.B h_{\mathrm{s} 1}(r)\right|_{G=0}+O\left(B^{2}\right),
$$

where the parabolic leading order term is simply the $G=0$ version of Eq. (28). Substitution of this expansion into Eq. (40) and solving the equation that arises at first order leads to the solution for $\left.h_{\mathrm{s} 1}(r)\right|_{G=0}$, namely

$$
\begin{aligned}
\left.h_{\mathrm{s} 1}(r)\right|_{G=0}= & \frac{d^{2}}{8(d-1)}\left\{\ln \left(\frac{d}{r^{2}-1+d}\right)\right. \\
& \left.+2\left(r^{2}-1\right)\left[1-(d-1) \ln \left(\frac{d}{d-1}\right)\right]\right\},
\end{aligned}
$$

and so to $O(B)$ the equilibrium drop apex height difference when $G=0$ is given by

$$
\left.\Delta H_{\mathrm{s}}\right|_{G=0}=\frac{B d^{2}}{8(d-1)}\left[(2 d-1) \ln \left(\frac{d}{d-1}\right)-2\right],
$$

and the corresponding equilibrium contact angle difference is

$$
\left.\Delta \theta_{\mathrm{s}}\right|_{G=0}=-\frac{B d}{4(d-1)}\left[(2 d-1)-2 d(d-1) \ln \left(\frac{d}{d-1}\right)\right] .
$$

Although the assumptions used to obtain Eqs. (43) and (44), namely that $B \ll 1$ and $G=0$, are questionable given the experimental value of $G=0.9457$ and values of $B$ up to $B=$ 14.0673, these expressions are still useful since they reveal the 


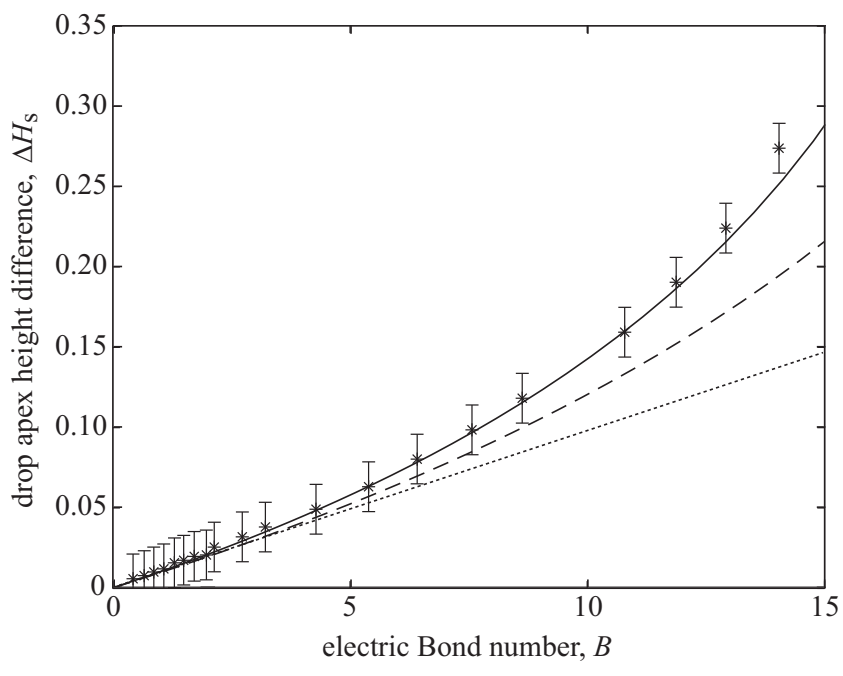

FIG. 3. The nondimensional equilibrium drop apex height difference $\Delta H_{\mathrm{s}}$ plotted as a function of the electric Bond number $B$, obtained experimentally (shown with stars) with error bars corresponding to a $95 \%$ confidence interval using one pixel as the standard error, using the approximate analytical solution valid for $B \ll 1$ and $G=0$ given by Eq. (49) (shown with a dotted line), obtained numerically from the model using the thin-film approximation (shown with a dashed line), and obtained numerically from the full model without using the thin-film approximation (shown with a solid line).

asymptotic dependencies of the solution on $d$. Specifically, they show that

$$
\begin{gathered}
\left.\Delta H_{\mathrm{s}}\right|_{G=0}=-\frac{B}{8} \frac{\ln (d-1)}{(d-1)}+O\left(\frac{1}{d-1}\right) \text { as } d \rightarrow 1^{+}, \\
\left.\Delta H_{\mathrm{s}}\right|_{G=0}=\frac{B}{48 d}+O\left(\frac{1}{d^{2}}\right) \text { as } d \rightarrow \infty, \\
\left.\Delta \theta_{\mathrm{s}}\right|_{G=0}=-\frac{B}{4}\left[\frac{1}{d-1}+2 \ln (d-1)\right]+O(1) \text { as } d \rightarrow 1^{+},
\end{gathered}
$$

$$
\left.\Delta \theta_{\mathrm{s}}\right|_{G=0}=-\frac{B}{12 d}+O\left(\frac{1}{d^{2}}\right) \text { as } d \rightarrow \infty,
$$

demonstrating a strengthening of the effect of the electric field as the upper electrode nears the apex of the drop, $d \rightarrow 1^{+}$, and a corresponding weakening of the effect as the plate separation increases, $d \rightarrow \infty$. For the present experimental values, namely $d_{\exp }=3.857$ and $\delta_{\exp }=0.0777$, from Eqs. (43) and (44) we obtain

$$
\left.\Delta H_{\mathrm{s}}\right|_{G=0}=0.00976 B \text { and }\left.\Delta \theta_{\mathrm{s}}\right|_{G=0}=-0.0337 B .
$$

Figure 3 shows the equilibrium drop apex height difference $\left.\Delta H_{\mathrm{s}}\right|_{G=0}$ plotted as a function of $B$ together with the corresponding experimental measurements. The experimental measurements were calculated by averaging the drop apex height difference over a 1 s time period, starting when the voltage had been applied for $2 \mathrm{~s}$, which corresponds to averaging between $t=0.364$ and $t=0.546$ in nondimensional time units. Because of the potential errors in measuring heights from pixellated images we have included error bars on the experimental data corresponding to a $95 \%$ confidence interval using one pixel as the standard error. We observe good agreement between the approximate analytical result (49) shown with a dotted line and the experimental results shown with stars for $B \lesssim 6.4$, which is surprisingly good in light of the approximations made, and corresponds to an approximate range of validity of $V_{\mathrm{m}} \lesssim 610 \mathrm{~V}$.

If we do not assume that either $B \ll 1$ or $G=0$, but continue to use the thin-film approximation, then we can solve the system using the finite element method package COMSOL [37] using a sufficiently fine mesh to ensure that the numerical error is two orders of magnitude smaller than the experimental errors. Equilibrium solutions are found by solving the partial differential equation (37) numerically with a constant applied voltage until equilibrium is reached (which we take to be the time at which the change in the drop apex height difference is at least two orders of magnitude smaller than the experimental error). The results obtained using this numerical method are shown in Fig. 3 using a dashed line. Again, good agreement is found between the experimental results and the predictions of the thin-film model, with agreement for a slightly larger range of $B$ than the approximate analytical solution (49). In particular, the thin-film solution lies within the experimental error bars for $B \lesssim 8.6$, corresponding to an approximate range of validity of $V_{\mathrm{m}} \lesssim 710 \mathrm{~V}$.

In equilibrium we can also use the numerical method developed by Corson et al. [10] to solve the full model without using the thin-film approximation, without any restrictions on the values of $B$ and $G$, i.e., solving the equations for the electric potential in the air gap (1) subject to the boundary conditions (2)-(4), the normal stress balance (9), the boundary conditions for the drop free surface (13), and the volume constraint (14). Full details of this numerical method are provided in Ref. [10], but, essentially, the method iteratively solves the electric potential equation for a fixed drop free surface shape and then updates the drop free surface shape by solving the normal stress balance using the calculated electric potential, the process being repeated until convergence. Using this method the drop apex height difference was calculated as a function of $B$ and is shown in Fig. 3 using a solid line. Very good agreement is obtained between the experimental results and these numerical results, indicating that the model is a very good representation of the experiments, at least for the range of voltages considered here.

\section{B. Dynamic solutions}

In the remainder of the present work we consider the dynamic response of the drop as the electric field is either abruptly applied or abruptly removed. Figure 4 shows the experimentally measured drop apex height difference $\Delta H$ (shown with circles) plotted as a function of time $t$ for four voltage pulses of different amplitudes $V_{\mathrm{m}}$ but a constant pulse duration of $T=1.82$ nondimensional time units, equivalent to $10 \mathrm{~s}$. Mathematically we model this form of the nondimensionalized applied voltage using $V(t)=H(t) H(T-t)$, 

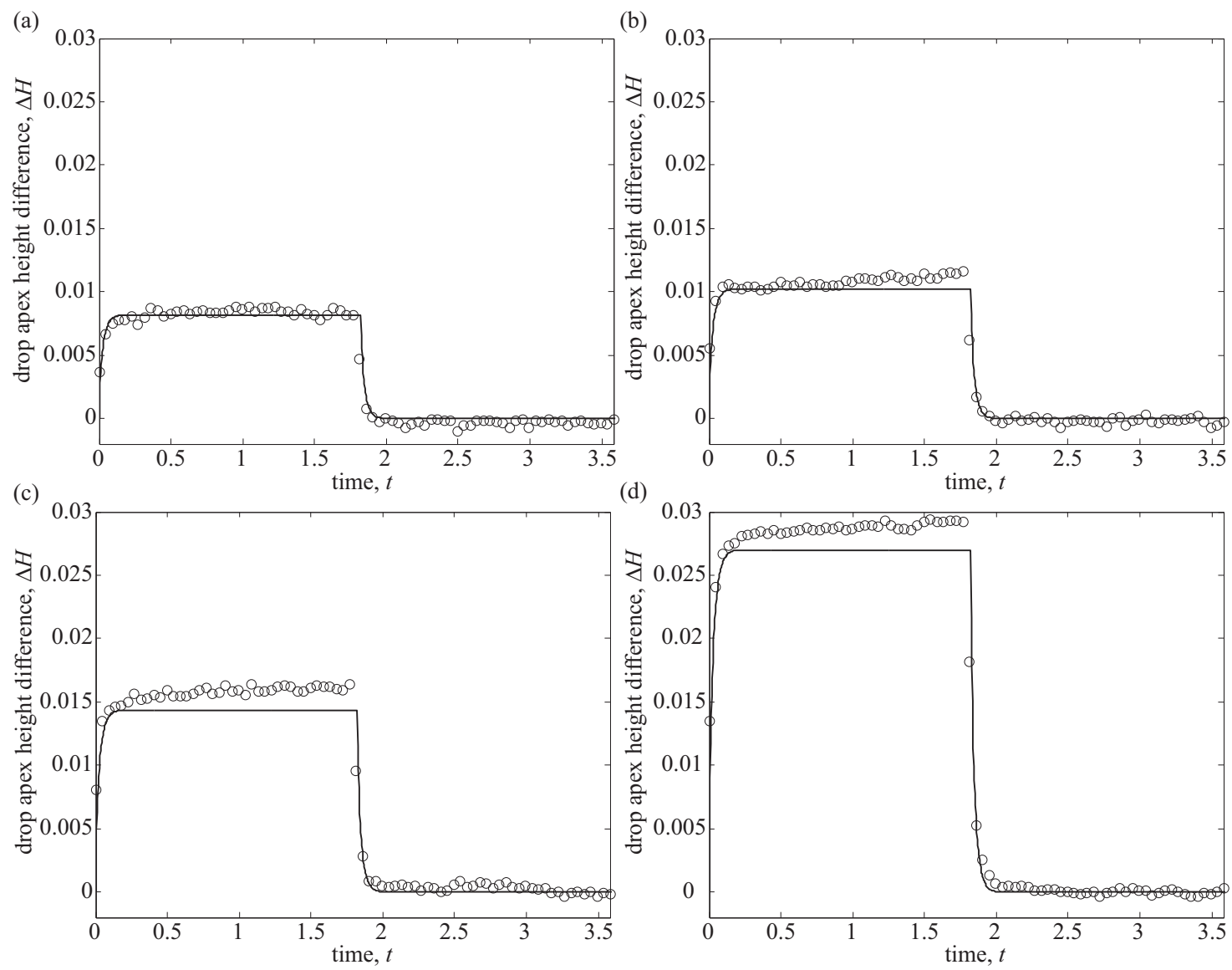

FIG. 4. The experimentally measured nondimensional drop apex height difference $\Delta H$ (shown with circles) plotted as a function of nondimensional time $t$ for voltage pulses with four different electric Bond numbers $B=0.86,1.08,1.50,2.73$, but a constant nondimensional pulse duration of $T=1.82$, along with the corresponding numerical solution of the thin-film equation (37) (shown with solid lines). For clarity, the experimental data is plotted using average values over 500 experimental times steps, although all of the analysis of the experimental data uses the complete data set.

where $H(\cdot)$ is the Heaviside function. The dynamic numerical solution of the thin-film equation (37) is shown with solid lines in Fig. 4. As previously shown in Fig. 3, Fig. 4 confirms that the equilibrium drop apex heights are captured reasonably well by the thin-film equation, certainly for small electric Bond numbers, but there is also evidence of some slow upwards drift in the experimental results when the voltage was applied. The cause of this slow drift in the drop apex height may be linked to aspects of the experimental geometry that were difficult to maintain, notably a pinned and circular contact line, and sufficient parallelism between the substrate and the upper electrode when the voltage was applied. We have used a voltage pulse duration that ensures that the change in $\Delta H$ due to this drift is always less than $10 \%$. It is also apparent from Fig. 4 that the transient behavior is captured reasonably well during both the switch-on and the switch-off phases.

In order to investigate further the transient behavior of the drop we now consider approximate solutions valid for short times after an abrupt change in the applied voltage.

Assuming that the system is in equilibrium with $h=h_{\mathrm{s}}(r)$ for $t \leqslant t_{0}$, an abrupt change in the applied voltage at time $t=t_{0}$ from $V_{0}$ to $V_{1}$ (where $t_{0}=0, V_{0}=0$ and $V_{1}=V_{\mathrm{m}}$ for switch on, and $t_{0}=T, V_{0}=V_{\mathrm{m}}$, and $V_{1}=0$ for switch off) can be modeled as a corresponding abrupt change in the electric Bond number from $B_{0}$ to $B_{1}$, with a resulting change to the drop free surface profile $h(r, t)$ for $t>t_{0}$. For short times after $t=t_{0}$ the drop free surface profile can be written as

$$
h(r, t)=h_{\mathrm{s}}(r)+\left(t-t_{0}\right) h_{1}(r)+O\left(\left(t-t_{0}\right)^{2}\right),
$$

and the solution for $h_{1}(r)$ can be found by expanding Eq. (40) in powers of $t-t_{0} \ll 1$ to give

$$
h_{1}(r)=-\left(B_{1}-B_{0}\right) \frac{1}{r} \frac{d}{d r}\left[\frac{r d^{2} h_{\mathrm{s}}^{3}}{3\left(d-h_{\mathrm{s}}\right)^{3}} \frac{d h_{\mathrm{s}}}{d r}\right] .
$$

The practical value of this equation depends on our ability to obtain $h_{\mathrm{s}}(r)$ from Eq. (40) and thus to determine $h_{1}(r)$ from Eq. (51). The drop apex height difference is then given by

$$
\Delta H(t)=\Delta H_{\mathrm{s}}+\left(t-t_{0}\right) h_{1}(0)+O\left(\left(t-t_{0}\right)^{2}\right),
$$

where $\Delta H_{\mathrm{s}}=h_{\mathrm{s}}(0)-1$ is the equilibrium drop apex height difference derived from the solution to Eq. (40). We now discuss a number of situations in which further analytical progress is possible.

\section{Switch on}

The simplest case is that of switch on, in which the initial state is the zero-field solution, so that the electric Bond number 
changes abruptly from $B_{0}=0$ to $B_{1}=B \neq 0$ at time $t=0$. In this case the initial drop shape is given by Eq. (28), so that $\Delta H_{\mathrm{s}}=0$, and using Eq. (51) we find the drop apex height difference to be

$$
\Delta H(t)=\frac{B G d^{2}}{3(d-1)^{3}\left(I_{0}(\sqrt{G})-1\right)} t+O\left(t^{2}\right) .
$$

Using the values of the physical properties of $[\mathrm{Bmim}]\left[\mathrm{BF}_{4}\right]$ and the physical dimensions of the experimental system described in Sec. III, we obtain the theoretical prediction $\Delta H(t)=0.802 B t+O\left(t^{2}\right)$.

In some instances gravitational effects can be small and it is therefore useful to consider the expansion of Eq. (53) for small values of $G$, namely

$$
\begin{aligned}
\Delta H(t)= & \frac{4 B d^{2}}{3(d-1)^{3}}\left(1-\frac{1}{16} G+\frac{5}{2304} G^{2}+\cdots+c_{n} G^{n}+\cdots\right) t \\
& +O\left(t^{2}\right) .
\end{aligned}
$$

The coefficients of $G^{n}$ in the bracketed expansion, denoted by $c_{n}$, are calculated using the recursion relation

$$
c_{0}=1, \quad c_{n}=-\sum_{k=0}^{n-1} \frac{c_{k}}{4^{n-k}[(n-k+1) !]^{2}},
$$

which was derived from Eq. (53) using the small argument expansion of the modified Bessel function [38, identity 9.6.12 on page 375], and using the Cauchy product for infinite series to determine the reciprocal of the series representation of the modified Bessel function.

Defining $\lambda$ to be the coefficient of $B t$ in the short-time expansion of the drop apex height difference (53), so that $\Delta H(t)=\lambda B t+O\left(t^{2}\right)$, Fig. 5 is a plot of $\lambda$ versus $G$ using the forms of $\lambda$ derived from Eq. (53) (shown with a solid line), the two-term truncation of Eq. (54) (shown with a dashed line) and the three-term truncation of Eq. (54) (shown with a dotted line). Figure 5 shows that the two-term expansion is reasonably accurate up to $G \approx 2$, and that the three-term expansion is reasonably accurate up to $G \approx 6$. In fact, as Fig. 5 shows, even though the experimental value of $G$ in the present work, $G_{\text {exp }}=0.9472$, is not particularly small, the truncated forms of Eq. (54) are still remarkably accurate. In particular, using the two-term truncation yields the approximate expression $\lambda \approx$ 0.800 , which agrees very well with the exact expression obtained from Eq. (53), namely $\lambda=0.802$. Consideration of the two- and three-term truncations to higher values of $G$ than $G_{\text {exp }}$ corresponds to systems with larger density and/or drop radius and/or smaller surface tension than in the present experiments.

We may also compute the dynamic numerical solution of the thin-film equation (37). Using an expression for the drop apex height difference of the form $\Delta H(t) \approx \lambda B t$ and fitting the numerical data for a sufficiently short period of time after $t=0$ we recover the appropriate value of $\lambda$, namely $\lambda=0.802$.

Guided by the form of the short-time expansion (53) we model the short-time experimental data by assuming that the drop apex height difference is of the form $\Delta H(t) \approx \Delta H_{0}+$ $\lambda B t$. Here we have allowed for the possibility of an initial offset $\Delta H_{0} \neq 0$ in the experimental results, in order to account for the possibility of any offsetting in the experimental data due to errors in the camera position and inaccuracies of measuring

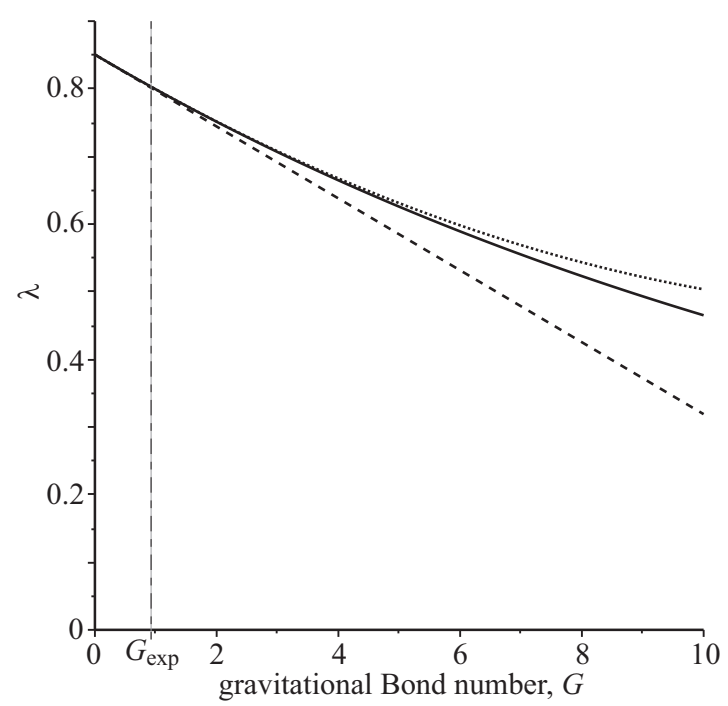

FIG. 5. Plot of $\lambda$, the coefficient of $B t$ in the short-time expansion of the nondimensional drop apex height difference $\Delta H=\lambda B t+$ $O\left(t^{2}\right)$ during switch on from $B_{0}=0$ to $B_{1}=B$ at time $t=0$, as a function of the gravitational Bond number $G$. The solid line represents the solution (53) and the dashed and dotted lines represent the two-term and three-term truncations of Eq. (54), respectively. The experimental value of $G$ in the present work, $G_{\exp }=0.9472$, is indicated with the vertical dashed line.

the equilibrium drop apex height. Then, for each voltage pulse, we fit the experimental data for a short period of time after $t=0$ to obtain the experimental value of $\lambda$. Figure 6(a) shows the experimental data for the drop apex height difference $\Delta H$ for short times after switch on plotted as a function of time. For clarity, the experimental data is plotted in Fig. 6(a) using average values over five experimental time steps and for only eleven voltages, corresponding to electric Bond numbers $B=$ $2.73,3.22,4.29,5.39,6.42,7.57,8.63,10.79,11.87,12.92$, 14.04 , although all of the analysis of the experimental data uses the complete data set. The experimental values of $\lambda$ calculated from the experimental data are plotted as a function of $B$ in Fig. 6(b), with the error bars corresponding to $95 \%$ confidence intervals. Figure 6(b) also shows the average value of $\lambda$ using all of the experimental data (shown with a solid line), namely $\lambda=0.471 \pm 0.087$ (95\% confidence interval). It is clear from Fig. 6(b) that there is significant uncertainty and scatter in the experimental values of $\lambda$ for low voltages. The average value of $\lambda$ using only the experimental data for values of $B$ satisfying $B \geqslant 4.29$ (shown with a dashed line) is somewhat higher, namely $\lambda=0.586 \pm 0.011$ ( $95 \%$ confidence interval). Given the challenging nature of the experiment, the approximations used in the theory, and the fact that we have not used any fitting parameters in our comparison of data with theory, this latter average value is in reasonably good agreement with the theoretical prediction of $\lambda=0.802$ obtained from Eq. (53).

\section{Switch off}

We now consider the case of switch off, in which the electric Bond number changes abruptly from the nonzero value $B_{0}=$ $B \neq 0$ to $B_{1}=0$ at time $t_{0}=T$. As we have seen in Sec. IV A, analytical progress in determining the equilibrium solution for 
(a)

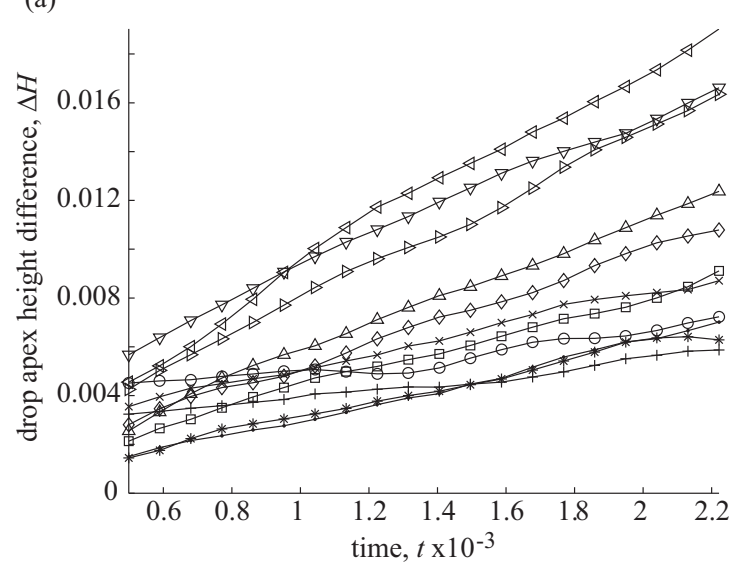

(b)

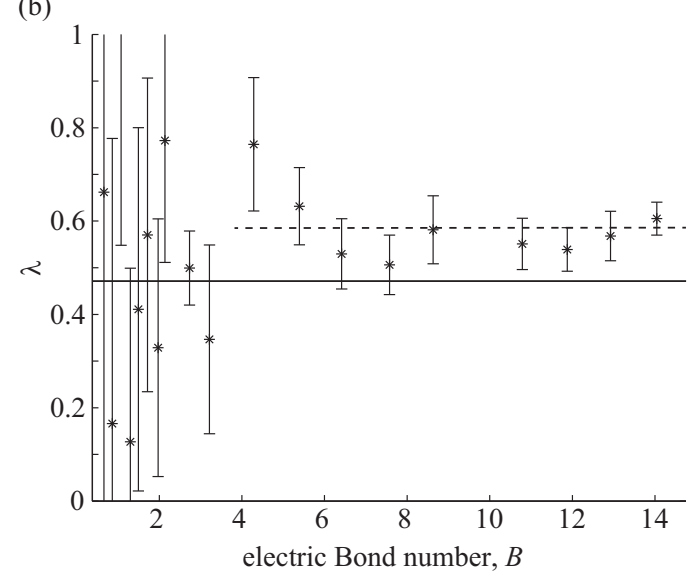

FIG. 6. Extraction of the switch-on timescale from the experimental data: (a) data for $\Delta H$ for short times after switch on, with larger electric Bond numbers $B$ corresponding to larger values of $\Delta H$, plotted as a function of time $t$. For clarity, the experimental data is plotted using average values over five experimental time steps and for only 11 voltages, corresponding to $B=2.73,3.22,4.29,5.39,6.42,7.57,8.63,10.79$, $11.87,12.92,14.04$, although all of the analysis of the experimental data uses the complete data set; (b) experimental values of $\lambda$ assuming that $\Delta H(t) \approx \Delta H_{0}+\lambda B t$ plotted as a function of $B$, together with the average value of $\lambda$ using all of the experimental data, namely $\lambda=0.471$ (shown with a solid line), and the average value of $\lambda$ using only the experimental data for values of $B$ satisfying $B \geqslant 4.29$, namely $\lambda=0.586$ (shown with a dashed line). The error bars on the values of $\lambda$ correspond to $95 \%$ confidence intervals.

the nonzero field case is possible only if $B \ll 1$ and $G=0$. We therefore consider the equilibrium drop free surface profile associated with these approximations given by Eqs. (41) and (42), so that the equilibrium drop apex height difference $\Delta H_{\mathrm{s}}$ is given by Eq. (43). For $t>T$ the solution for $h_{1}(r)$ can be found from Eq. (51). Setting $r=0$ in this solution then allows the expression for the dynamic drop apex height difference when $G=0$ to be found, namely

$$
\begin{aligned}
\left.\Delta H(t)\right|_{G=0}= & \frac{B d^{2}}{8(d-1)}\left[(2 d-1) \ln \left(\frac{d}{d-1}\right)-2\right] \\
& -\frac{4 B d^{2}}{3(d-1)^{3}}\left\{1+\frac{B d^{2}}{8(d-1)^{2}}\left[\left(8 d^{2}-7 d+2\right) \ln \left(\frac{d}{d-1}\right)-8 d+3\right]\right\}(t-T)-O\left[(t-T)^{2}\right] .
\end{aligned}
$$

We note that the leading (but not higher) order terms in the switch-on and switch-off expressions, given by Eqs. (54) and (56), respectively, are the same. For the present experimental values, Eq. (56) leads to the theoretical prediction

$$
\begin{aligned}
\left.\Delta H(t)\right|_{G=0}= & 0.00976 B-(0.851+0.0695 B) B(t-T) \\
& +O\left((t-T)^{2}\right) .
\end{aligned}
$$

In a similar way to in the switch-on case, we can compute the dynamic numerical solution of the thin-film equation (37) when the voltage is abruptly switched off at time $t=T$, and then fit the numerical data to an expression for the drop apex height difference of the form $\Delta H(t) \approx \Delta H_{\mathrm{s}}+\lambda B(t-T)$ for a sufficiently short period of time after $t=T$. In the case $G=0$ we recover the appropriate value of $\lambda$, namely $\lambda=-(0.851+0.0695 B)$. Figure 7 shows the numerically determined value of $\lambda$ as a function of $B$ for different values of $G$, and shows that for increasing values of $G$ the value of $|\lambda|$ decreases, so that the decay of $\Delta H(t)$ is slower. This result, namely that a stronger gravitational effect leads to a slower relaxation to the zero-field equilibrium state, may at first seem surprising. However, for an increased value of $G$ the equilibrium value of the non-zero field drop apex height difference $\Delta H_{\mathrm{s}}$ is reduced, leading to a slower relaxation to
$\Delta H=0$ when the voltage was switched off. Figure 7 also shows that the variation of $\lambda$ with $B$ (i.e., the gradient $d \lambda / d B$ ) remains approximately constant.

In a similar way to in the switch-on case, we also consider an expression for the experimental data for the drop apex height difference for the switch-off case of the form $\Delta H(t) \approx \Delta H_{\mathrm{s}}+\Delta H_{0}+\lambda B(t-T)$, where $\Delta H_{\mathrm{s}}$ is the value of the equilibrium drop apex height difference obtained experimentally previously shown in Fig. 3 (shown with stars), and we have again allowed for the possibility of an offset $\Delta H_{0} \neq 0$ from the equilibrium drop apex height difference when the voltage was switched off at $t=T$.

Figure 8(a) shows the experimental data for the drop apex height difference $\Delta H$ for short times after switch off plotted as a function of time. Figure 8(b) shows the experimental values of $\lambda$, calculated from the best fit for the experimental data assuming it to be of the form $\Delta H(t) \approx \Delta H_{\mathrm{s}}+\Delta H_{0}+$ $\lambda B(t-T)$, for all of the experimental values of $B$. Figure 8(b) also shows the best linear fit for $\lambda$ as a function of $B$ (shown with a solid line), namely $\lambda=-(0.378+0.090 B) \pm$ $(0.081+0.0000160 B)(95 \%$ confidence interval $)$.

For the experimental value of $G$, namely $G_{\exp }=0.9472$, our numerically determined expression for the dependence of $\lambda$ on $B$ is $\lambda=-(0.732+0.052 B)$. The leading term in 


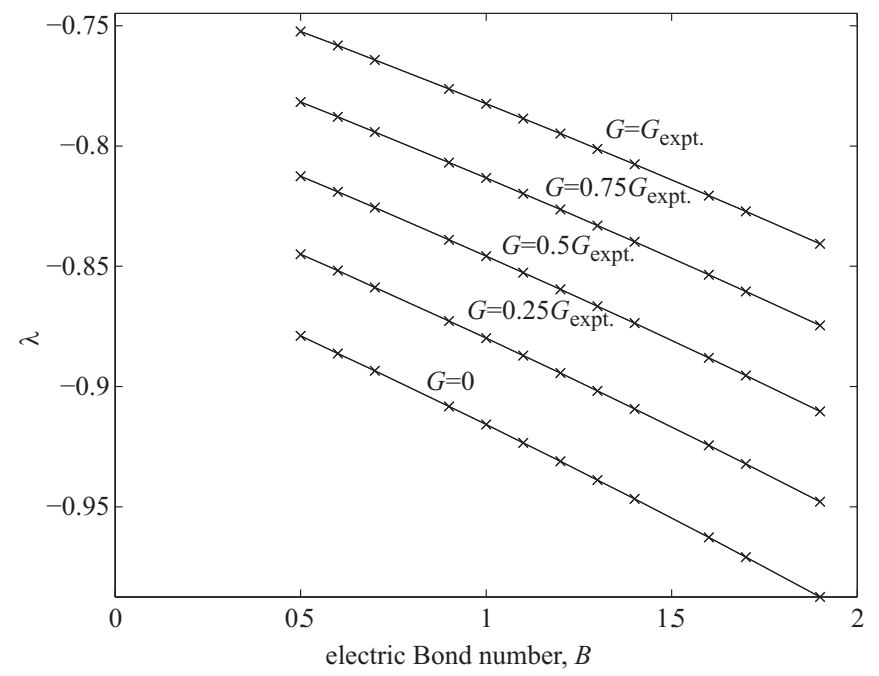

FIG. 7. Plot of $\lambda$, the coefficient of $B t$ in the short-time expansion of the nondimensional drop apex height difference $\Delta H=\Delta H_{\mathrm{s}}+$ $\lambda B(t-T)+O\left((t-T)^{2}\right)$ during switch off from $B_{0}=B$ to $B_{1}=0$ at time $t=T$, as a function of the electric Bond number $B$, determined from the dynamic numerical solution of the thin-film equation (37), and for values of the gravitational Bond number $G$ from zero to the experimental value $G_{\exp }=0.9472$.

this expression is almost a factor of two larger than the experimentally determined expression for $\lambda$, namely $\lambda=$ -0.378 , but, as in the switch-on case, given the challenging nature of the experiment, the approximations used in our theory, and the fact that we have not used any fitting parameters in our comparison of data with theory, this is still quite an encouraging level of agreement.

(a)

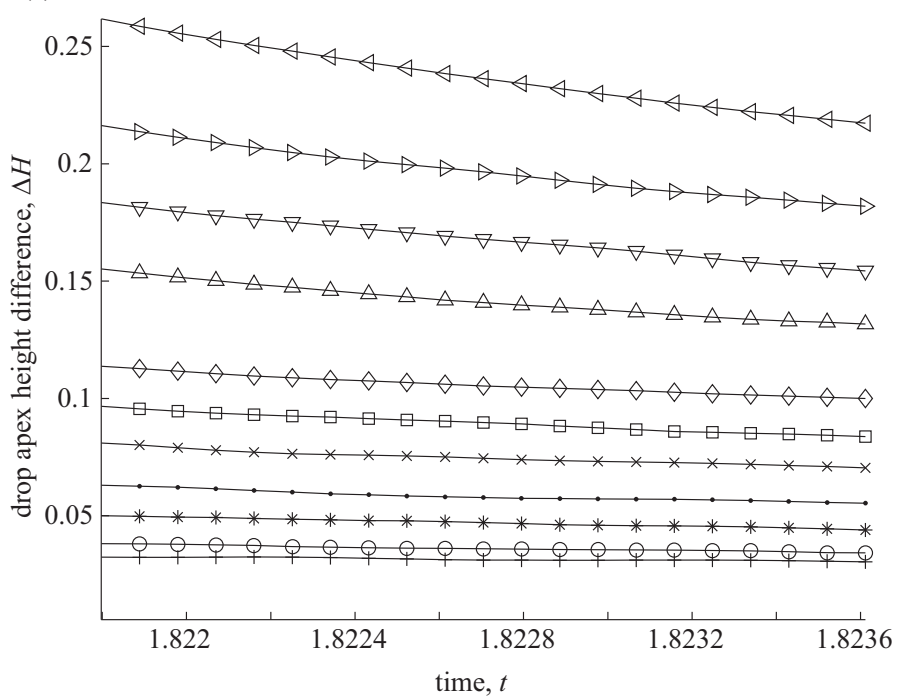

\section{CONCLUSIONS}

In the present work we considered the dynamic response of a thin sessile drop of conductive liquid lying within a parallel-plate capacitor. In the static case, we found very good agreement between the numerical solution of the full theoretical model and experimental results when a DC voltage is applied. When a thin-film approximation is employed, the numerical solution of the simplified model agrees with experimental results for a restricted range of electric Bond numbers $B$. A further simplification of the model, namely that $B \ll 1$ and $G=0$, leads to analytical results that are valid for a slightly smaller range of $B$, which reveal the asymptotic dependencies of the solution on the separation of the capacitor plates. In the case of the dynamic response to either the abrupt application or the abrupt removal of the electric field, the numerical and analytical approaches give reasonably good agreement for switch-on behavior and less good, but still encouraging, agreement for switch-off behavior. Further experiments, and possibly an appropriately refined theoretical model, are required to understand better the effects of charge redistribution and lack of rotational symmetry on the behavior of the drop.

The reasonable agreement between the experimental results and the analytical results lead us to suggest that the expressions for the drop free surface and drop apex height difference may be used predictively in other situations. In the equilibrium case, the drop free surface and the drop apex height difference are predicted to obey Eqs. (42) and (43), respectively. In the switch-on case, the dynamic drop apex height difference is predicted to obey Eq. (53), with the two-term truncation of Eq. (54) providing a simpler form. In the switch-off case, the dynamic drop apex height difference is predicted to obey

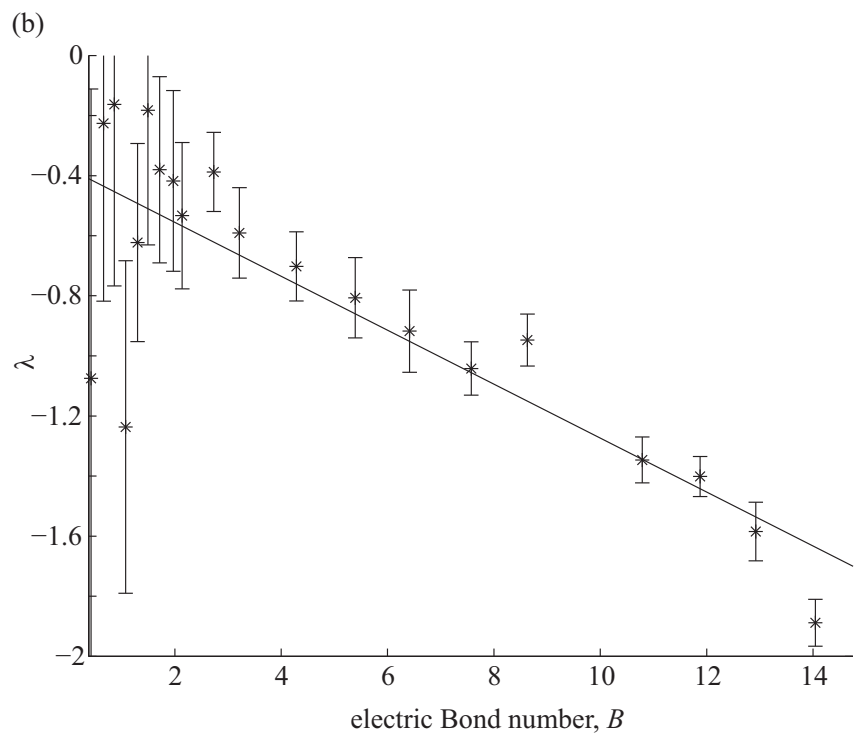

FIG. 8. Extraction of the switch-off timescale from the experimental data: (a) data for $\Delta H$ for short times after switch off, with larger electric Bond numbers $B$ corresponding to larger values of $\Delta H$, plotted as a function of time $t$. For clarity, the experimental data is plotted using average values over 5 experimental time steps and for only 11 voltages, corresponding to $B=2.73,3.22,4.29$, $5.39,6.42,7.57,8.63,10.79,11.87,12.92,14.04$, although all of the analysis of the experimental data uses the complete data set; (b) experimental values of $\lambda$ assuming that $\Delta H \approx \Delta H_{\mathrm{s}}+\Delta H_{0}+\lambda B(t-T)$ plotted as a function of $B$, together with the best linear fit for $\lambda$ as a function of $B$, namely $\lambda=-(0.378+0.090 B)$ (shown with a solid line). The error bars on the values of $\lambda$ correspond to $95 \%$ confidence intervals. 
Eq. (56). These analytical expressions can be used with some confidence to predict the behavior for different geometrical parameters and/or for liquids with different properties from those used in the experimental results presented in this paper, i.e., for drops of different size, for different capacitor air-gap sizes, and for fluids (either the drop liquid or the passive gas) with different densities, surface tensions or permittivities.

Research data associated with this paper is available through Ref. [39].

\section{ACKNOWLEDGMENTS}

This work was supported by the United Kingdom Engineering and Physical Sciences Research Council (EPSRC) via Grants No. EP/J009865/1 and No. EP/J009873/1. S.K.W. was also supported by Leverhulme Trust Research Fellowship Grant No. RF-2013-355. We gratefully acknowledge the advice given to us by Dr. Ian C. Sage (Nottingham Trent University) on the design of the present experiments and the choice of materials used.
[1] J. W. Swan, Stress and other effects produced in resin and in a viscid compound of resin and oil by electrification, Proc. R. Soc. Lond. 62, 38 (1897).

[2] K. J. Cheng and M. J. Miksis, Shape and stability of a drop on a conducting plane in an electric field, PhysicoChem. Hydrodynam. 11, 9 (1989).

[3] O. A. Basaran and L. E. Scriven, Axisymmetric shapes and stability of pendant and sessile drops in an electric field, J. Coll. Int. Sci. 140, 10 (1990).

[4] F. K. Wohlhuter and O. A. Basaran, Shapes and stability of pendant and sessile dielectric drops in an electric field, J. Fluid Mech. 235, 481 (1992).

[5] B. Berge and J. Peseux, Variable focal lens controlled by an external voltage: An application of electrowetting, Euro. Phys. J. E 3, 159 (2000).

[6] C. Quilliet and B. Berge, Electrowetting: A recent outbreak, Curr. Opin. Coll. Int. Sci. 6, 34 (2001).

[7] S. N. Reznik, A. L. Yarin, A. Theron, and E. Zussman, Transient and steady shapes of droplets attached to a surface in a strong electric field, J. Fluid Mech. 516, 349 (2004).

[8] F. Mugele and J.-C. Baret, Electrowetting: From basics to applications, J. Phys.: Condens. Matter 17, R705 (2005).

[9] L. Chen and E. Bonaccurso, Electrowetting-from statics to dynamics, Adv. Coll. Int. Sci. 210, 2 (2014).

[10] L. T. Corson, C. Tsakonas, B. R. Duffy, N. J. Mottram, I. C. Sage, C. V. Brown, and S. K. Wilson, Deformation of a nearly hemispherical conducting drop due to an electric field: Theory and experiment, Phys. Fluids 26, 122106 (2014).

[11] C. Tsakonas, L. T. Corson, I. C. Sage, and C. V. Brown, Electric field induced deformation of hemispherical sessile drops of ionic liquid, J. Electrostat. 72, 437 (2014).

[12] R. V. Craster and O. K. Matar, Dynamics and stability of thin liquid films, Rev. Mod. Phys. 81, 1131 (2009).

[13] E. Schaffer, T. Thurn-Albrecht, T. P. Russell, and U. Steiner, Electrically induced structure formation and pattern transfer, Nature 403, 874 (2000).

[14] E. Schaffer, T. Thurn-Albrecht, T. P. Russell, and U. Steiner, Electrohydrodynamic instabilities in polymer films, Europhys. Lett. 53, 518 (2001).

[15] M. D. Morariu, N. E. Voicu, E. Schäffer, Z. Lin, T. P. Russell, and U. Steiner, Hierarchical structure formation and pattern replication induced by an electric field, Nature Mat. 2, 48 (2003).

[16] A. Klingner, S. Herminghaus, and F. Mugele, Self-excited oscillatory dynamics of capillary bridges in electric fields, Appl. Phys. Lett. 82, 4187 (2003).
[17] A. Klingner, J. Buehrle, and F. Mugele, Capillary bridges in electric fields, Langmuir 20, 6770 (2004).

[18] L. F. Pease and W. B. Russel, Electrostatically induced submicron patterning of thin perfect and leaky dielectric films: A generalized linear stability analysis, J. Chem. Phys. 118, 3790 (2003).

[19] R. V. Craster and O. K. Matar, Electrically induced pattern formation in thin leaky dielectric films, Phys. Fluids 17, 032104 (2005).

[20] D. Merkt, A. Pototsky, M. Bestehorn, and U. Thiele, Long-wave theory of bounded two-layer films with a free liquid-liquid interface: Short- and long-time evolution, Phys. Fluids 17, 064104 (2005).

[21] R. Verma, A. Sharma, K. Kargupta, and J. Bhaumik, Electric field induced instability and pattern formation in thin liquid films, Langmuir 21, 3710 (2005).

[22] N. Wu, L. F. Pease, and W. B. Russel, Electric-field-induced patterns in thin polymer films: Weakly nonlinear and fully nonlinear evolution, Langmuir 21, 12290 (2005).

[23] D. Tseluiko and D. T. Papageorgiou, Nonlinear dynamics of electrified thin liquid films, SIAM J. Appl. Math. 67, 1310 (2007).

[24] H. K. Yeoh, Q. Xu, and O. A. Basaran, Equilibrium shapes and stability of a liquid film subjected to a nonuniform electric field, Phys. Fluids 19, 114111 (2007).

[25] S. Manigandan, S. Majumder, A. Suresh, S. Ganguly, K. Kargupta, and D. Banerjee, Electric field induced dewetting and pattern formation in thin conducting polymer film, Sensors Actuators B: Chem. 144, 170 (2010).

[26] C. W. J. Berendsen, C. J. Kuijpers, J. C. H. Zeegers, and A. A. Darhuber, Dielectrophoretic deformation of thin liquid films induced by surface charge patterns on dielectric substrates, Soft Matter 9, 4900 (2013).

[27] A. Ramkrishnan and S. Kumar, Electrohydrodynamic deformation of thin liquid films near surfaces with topography, Phys. Fluids 26, 122110 (2014).

[28] A. Corbett and S. Kumar, Combined thermal and electrohydrodynamic patterning of thin liquid films, J. Eng. Math. 94, 81 (2015).

[29] L. Y. Yeo, R. V. Craster, and O. K. Matar, Drop manipulation and surgery using electric fields, J. Coll. Int. Sci. 306, 368 (2007).

[30] Y. Chauvin, L. Mussmann, and H. Olivier, A novel class of versatile solvents for two-phase catalysis: Hydrogenation, isomerization, and hydroformylation of alkenes catalyzed by rhodium complexes in liquid 1, 3-dialkylimidazolium salts, Angew. Chem. Int. Ed. Engl. 34, 2698 (1995). 
[31] P. A. Z. Suarez, J. E. L. Dullius, S. Einloft, R. F. De Souza, and J. Dupont, The use of new ionic liquids in two-phase catalytic hydrogenation reaction by rhodium complexes, Polyhedron 15, 1217 (1996).

[32] Z. Fei and P. J. Dyson, The making of iLiquids - the chemist's equivalent of the iPhone, Chem. Commun. 49, 2594 (2013).

[33] O. Ciocirlan, O. Croitoru, and O. Iulian, Densities and viscosities for binary mixtures of 1-butyl-3-methylimidazolium tetrafluoroborate ionic liquid with molecular solvents, J. Chem. Eng. Data 56, 1526 (2011).

[34] Y.-H. Yu, A. N. Soriano, and M.-H. Li, Heat capacity and electrical conductivity of aqueous mixtures of $\left[\mathrm{Bmim}^{-}\right]\left[\mathrm{BF}_{4}\right]$ and $[\mathrm{Bmim}]\left[\mathrm{PF}_{6}\right]$, J. Taiwan Inst. Chem. Engineers 40, 205 (2009).
[35] J. Sangoro, C. Iacob, A. Serghei, S. Naumov, P. Galvosas, J. Kärger, C. Wespe, F. Bordusa, A. Stoppa, J. Hunger, R. Buchner, and F. Kremer, Electrical conductivity and translational diffusion in the 1-butyl-3-methylimidazolium tetrafluoroborate ionic liquid, J. Chem. Phys. 128, 214509 (2008).

[36] A. W. Adamson and A. P. Gast, Physical Chemistry of Surfaces, 6th ed. (John Wiley \& Sons Inc., New York, 1997).

[37] COMSOL Multiphysics, version 4.3b, COMSOL Inc. (2013).

[38] M. Abramowitz and I. Stegun, Handbook of Mathematical Functions, 10th printing of 1964 edition with corrections, United States National Bureau of Standards, Applied Mathematics Series 55 (1972).

[39] University of Strathclyde KnowledgeBase Data Set http://dx. doi.org/10.15129/1d0dc167-0e9d-44b9-96d0-b44614d4ac22. 